\title{
Prostatite chronique, syndrome douloureux pelvien chronique et dysfonctions sexuelles
}

\author{
Dominique DELAVIERRE
}

Service Urologie - Andrologie, CHR La Source, Orléans

RESUME

En 1995 le NIH (National Institutes of Health, USA) a proposé une nouvelle classification des prostatites chroniques (PC) avec pour objectif de sortir cette pathologie du cadre strict de la prostate et de la recentrer sur la notion de douleur pelvienne. Cette classification a introduit la terminologie de syndrome douloureux pelvien chronique (SDPC). La définition de ce syndrome précise qu'aux douleurs s'associent parfois des troubles sexuels.

De nombreuses enquêtes ont montré des prévalences de PC/SDPC non négligeables et confirmé leur impact sur la qualité de vie, mais actuellement les données épidémiologiques concernant les liens entre PC/SDPC et sexualité sont encore limitées.

La physiopathologie des dysfonctions sexuelles associées aux PC/SDPC (altération du désir, dysfonction érectile et éjaculation prématurée) reste par ailleurs imprécise. Une implication psychologique est très probable, mais beaucoup d'incertitudes persistent sur d'autres mécanismes éventuellement en cause.

Mots-clés : prostatite, douleur pelvienne, dysfonction sexuelle, dysfonction érectile, éjaculation prématurée

\section{INTRODUCTION}

Depuis les travaux de Drach et al. publiés en 1978 [6], les prostatites étaient regroupées en prostatites aiguës, chroniques, bactériennes ou non, et prostatodynies. Cette classification reposait sur la clinique et l'épreuve microbiologique de Meares et Stamey (analyse fractionnée et comparative des urines et des sécrétions prostatiques obtenues par massage prostatique) décrite en 1967 [13].

En 1995, le NIH (National institutes of health, USA) et un groupe de travail sur la prostatite chronique (PC) (the chronic prostatitis collaborative research network study group) ont proposé une nouvelle classification avec pour objectif de sortir cette pathologie du cadre strict de la prostate et de la recentrer sur la notion de douleur pelvienne [16]. Toujours basée sur la clinique et la microbiologie, cette classification distingue la prostatite aiguë, la PC bactérienne, le syndrome douloureux pelvien chronique (SDPC), inflammatoire ou non, et une nouvelle entité, la prostatite inflammatoire asymptomatique. Le Tableau 1 détaille la définition des différentes catégories de prostatites. Celle du SDPC précise qu'aux douleurs s'associent parfois des troubles sexuels.

Correspondance :

Dr Dominique DELAVIERRE - Service Urologie Andrologie, CHR La Source, BP 6709, 45067 Orléans cedex 2 - Tel 0238514626 - Fax 0238514159 -

Email delav.uro@wanadoo.fr 
Tableau 1 : Définition et classification des prostatites [16].

- Prostatite aiguë bactérienne : infection aiguë de la prostate (catégorie I).

- Prostatite chronique bactérienne : infection chronique ou récidivante de la prostate par des agents bactériens (catégorie II).

- Syndrôme douloureux pelvien chronique (catégorie III) : douleur pelvienne génito-urinaire sans bactéries évoluant depuis au moins 3 mois parfois associée à des troubles mictionnels et sexuels, inflammatoire (IIIA) ou non (IIIB) selon la présence ou non de leucocytes dans les sécrétions prostatiques, les urines recueillies après massage prostatique ou le sperme.

- Prostatite inflammatoire asymptomatique (catégorie IV) : inflammation histologique ou présence de leucocytes dans les sécrétions prostatiques, les urines recueillies après massage prostatique ou le sperme.

En 1999, dans le prolongement de cette classification, le NIH et le groupe de travail sur la PC (Tableau 2) ont proposé un score symptomatique (NIH-CPSI : NIH chronic prostatitis symptom index) utilisant 9 questions concernant la dernière semaine écoulée : 4 sur la douleur (localisation, sévérité, fréquence), 2 sur les troubles mictionnels (irritatifs et obstructifs) et 3 sur la qualité de vie (conséquences des symptômes sur la vie quotidienne) [11]. Ce questionnaire ne comporte toutefois aucune question sur la sexualité.

De nombreuses enquêtes épidémiologiques effectuées en Amérique du Nord, en Europe et en Asie ont montré des prévalences de PC/SDPC non négligeables dans la population masculine (Tableau 3 ) et souligné l'impact de cette pathologie sur la qualité de vie, la fonction sexuelle et la santé mentale $[2-5,9,14,17,19-22,25]$.

\section{PC/SDPC ET SEXUALITE}

Plusieurs auteurs ont spécifiquement étudié les liens entre PC/SDPC et sexualité.

\section{Prévalence des dysfonctions sexuelles lors de prostatite chronique et de syndrome douloureux pelvien chronique}

Dès 1981 Keltikangas-Jarvinen et al. (Finlande) [8] notent des difficultés psychologiques chez $80 \%$ de 42 hommes présentant une PC : anxiété, dépression, fragilité affective, perte d'identité masculine et altération du désir sexuel.

En 1996 Berghuis et al. (USA) [1] comparent 51 patients présentant une $\mathrm{PC}$ à un groupe contrôle de 34 hommes. Quatre vingt cinq pour cent des patients présentant une PC ont réduit leurs contacts sexuels. Cette pathologie a perturbé, voire entraîné la rupture d'une relation chez $67 \%$ d'entre eux, et $43 \%$ estiment qu'elle est un frein à une nouvelle relation. Stress psychologique et dépression sont plus fréquents lors de PC.

En 2001 Screponi et al. (Italie) [23] évaluent 26 hommes d'âge moyen 43,8 ans présentant une $P C$, symptomatique ou non. Soixante et un pour cent signalent une éjaculation prématurée *.

En 2001 également, Mehik et al. (Finlande) [15] évaluent 261 hommes présentant une prostatite. Pendant les périodes symptomatiques $42,5 \%$ signalent une dysfonction érectile et $24,1 \%$ une altération du désir sexuel. Ces hommes ressentent un stress psychologique et sont volontiers nerveux, occupés, méticuleux avec des difficultés conjugales et relationnelles.

En 2002 Tan et al. (Singapour) [25] évaluent 1087 hommes de 21 à 70 ans : $2,7 \%$ présentent des symptômes évocateurs de prostatite, et leur score IIEF est significativement plus mauvais que celui des hommes sans ces symptômes.

En 2004 Liang et al. (Chine) [10] étudient 2498 patients âgés de 20 à 59 ans présentant une $\mathrm{PC}$ (évaluation par le questionnaire NIH-CPSI) : $21,8 \%$ présentent des douleurs sexuelles. 1768 sont éligibles pour l'évaluation de la fonction sexuelle (questionnaire IIEF) : $49 \%$ signalent une dysfonction sexuelle dont $26,2 \%$ une éjaculation prématurée ${ }^{\star}, 15 \%$ une dysfonction érectile (mais les patients présentant certains facteurs de risque - diabète, HTA, maladies psychiatriques, cardiopathies, médicaments - ont été exclus) et $7,7 \%$ les deux associées.

En 2005 Nickel [18] et les membres de l'enquête internationale ALF-ONE ( $A L F-O N E$ study group) étudient 3700 hommes présentant des troubles du bas appareil urinaire (TUBA) en rapport avec une hypertrophie bénigne de la prostate. Le questionnaire utilisé est le DAN-PSSsex (danish prostate symptom score sexual function questionnaire) qui évalue la rigidité de l'érection, la présence de douleurs ou d'une gêne lors de l'éjaculation et le volume de l'éjaculât : $18,6 \%$ des hommes présentent des douleurs ou une gêne lors de l'éjaculation (considérées comme des symptômes de prostatite) et $88 \%$ estiment que c'est un problème. Dans ce groupe, $72 \%$ présentent une altération de la rigidité de l'érection (contre $57 \%$ en l'absence de douleurs ou de gêne lors de l'éjaculation), $75 \%$ une hypospermie (contre $56 \%$ ) et $12 \%$ signalent des antécédents d'infection des voies urinaires (contre $7 \%$ ). Dans ce groupe, les patients sont également plus jeunes et leurs TUBA plus sévères.

En 2005 Lutz et al. (USA) [12] étudient 1764 hommes 


\section{Pain or Discomfort}

1. In the last week, have you experienced any pain or discomfort in the following areas?

Yes No ( 1 or 0 )

a. Area between rectum and testicles (perineum)

b. Testicles

c. Tip of the penis (not related to urination)

d. Below your waist, in your pubic or bladder area

2. In the last week, have you experienced:

Yes No ( 1 or 0 )

a. Pain or burning during urination ?

b. Pain or discomfort during or after sexual climax (ejaculation)?

3. How often have you had pain or discomfort in any of these areas over the last week?

0 Never

1 Rarely

2 Sometimes

3 Often

4 Usually

5 Always

4. Which number best describes your AVERAGE pain or discomfort on the days that you had it, over the last week?

012345678910

0 NO PAIN

10 AS PAIN BAD AS YOU CAN IMAGINE

\section{Urination}

5. How often have you had a sensation of not emptying your bladder completely after you finished urinating, over the last week ?

0 Not at all

1 Less than 1 time in 5

2 Less than half the time

3 About half the time

4 More than half the time

5 Almost always

6. How often have you had to urinate again less than two hours after you finished urinating, over the last week?
0 Not at all

1 Less than 1 time in 5

2 Less than half the time

3 About half the time

4 More than half the time

5 Almost always

Impact of Symptoms

7. How much have your symptoms kept you from doing the kinds of things you would usually do, over the last week?

0 None

1 Only a little

2 Some

$3 \mathrm{~A}$ lot

8. How much did you think about your symptoms, over the last week?

0 None

1 Only a little

2 Some

$3 \mathrm{~A}$ lot

\section{Quality of Life}

9. If you were to spend the rest of your life with your symptoms just the way they have been during the last week, how would you feel about that ?

0 Delighted

1 Pleased

2 Mostly satisfied

3 Mixed (about equally satisfied and dissatisfied)

4 Mostly dissatisfied

5 Unhappy

6 Terrible

Scoring the NIH-Chronic Prostatitis Symptom Index Domains

Pain : Total of items 1a, 1b, 1c,1d, 2a, 2b, 3, and 4 $=$

Urinary Symptoms : Total of items 5 and $6=$ Quality of Life Impact : Total of items 7, 8, and $9=$ 
Tableau 3 : Epidémiologie de la prostatite chronique et du syndrome douloureux pelvien chronique de l'homme.

\begin{tabular}{|c|c|c|c|c|}
\hline Auteur (Année) & Pays & Nombre d'hommes & Age (ans) & $\begin{array}{l}\text { Prévalence des } \\
\text { prostatites } \\
\text { ou de symptômes } \\
\text { évocateurs }\end{array}$ \\
\hline Roberts (1998) [22] & USA & 2113 & $40 / 79$ & $\begin{array}{l}8,8 \% \text { aiguës, chroniques } \\
\text { ou non préciséées }\end{array}$ \\
\hline Collins (1998) [4] & USA & 58955 visites & $\geq 18$ & $\begin{array}{c}8 \% \text { en urologie } \\
1 \% \text { en médecine générale }\end{array}$ \\
\hline Mehik (2000) [14] & Finlande & 1832 & $20 / 59$ & $\begin{array}{c}14,2 \% \\
\text { incidence }=37,8 / 10000 / \mathrm{an}\end{array}$ \\
\hline Nickel (2001) [17] & Canada & 868 & $20 / 74$ & $9,7 \%$ \\
\hline $\mathrm{Ku}(2001)$ [9] & Corée du sud & 16321 & 20 & $6 \%$ \\
\hline Collins (2002) [3] & USA & $\begin{array}{c}31681 \\
\text { professionnels } \\
\text { de santé }\end{array}$ & & $16 \%$ \\
\hline Roberts (2002) [21] & USA & 1541 & $40 / 79$ & $\begin{array}{c}12 \% \text { au moins } 1 \text { douleur } \\
\text { urogénitale } \\
2,2 \% \text { symptômes de } \\
\text { prostatites } \\
\end{array}$ \\
\hline $\operatorname{Tan}(2002)$ [25] & Singapour & 1087 & $21 / 70$ & $2,7 \%$ \\
\hline Cheah (2002) [2] & Malaisie & 3147 & $20 / 50$ & $8,7 \%$ \\
\hline Rizzo (2003) [20] & Italie & 8503 & & $\begin{array}{c}12,8 \% \\
\text { aiguës ou chroniques }\end{array}$ \\
\hline Nickel (2005) [19] & Canada & 8712 & & $2,7 \%$ \\
\hline Daniels (2005) [5] & USA & 5821 & $\geq 65$ & $\begin{array}{l}25 \% \text { aiguës ou chroniques } \\
\text { (antécédents ou en cours) }\end{array}$ \\
\hline
\end{tabular}

de plus de 40 ans (moyenne 61,6 ans) ayant une partenaire sexuelle régulière. II évalue les douleurs urogénitales (questionnaire NIH-CPSI) et la fonction sexuelle (questionnaire BMSFI). Les douleurs testiculaires sont associées significativement avec une altération du désir et de la satisfaction sexuels après ajustement sur l'âge. De même un score "douleur" supérieur ou égal à 4 sur le NIH-CPSI est associé significativement à une altération du désir sexuel et de l'éjaculation.

En 2005 Gonen et al. (Turquie) [7] comparent 66 hommes de 21 à 55 ans présentant un SDPC (évaluation par le questionnaire $\mathrm{NIH}-\mathrm{CPSI}$ ) à un groupe contrôle de 30 hommes. Cinquante et un $(77,3 \%)$ signalent une éjaculation prématuré $e^{*}$ et $10(15,2 \%)$ une éjaculation prématurée et une dysfonction érectile (évaluée par le questionnaire IIEF) associées. La différence avec le groupe contrôle est significative. II n'y a pas de lien entre les dysfonctions sexuelles et l'âge des patients, ni avec l'intensité ou l'ancienneté des symptômes du
SDPC. Le pharmaco-écho-doppler pénien et le bilan hormonal effectués chez les 10 patients présentant une DE sont normaux et les auteurs concluent à l'origine psychologique de la DE.

\section{Prévalence de la prostatite chronique lors d'éjaculation prématurée}

Deux travaux ont étudié la prévalence de la PC lors d'éjaculation prématurée avec des résultats très comparables [23, 24].

En 2001 Screponi et al. (Italie) [23] comparent 46 hommes de 18 à 68 ans présentant une éjaculation prématurée* à un groupe contrôle de 30 hommes. La dysfonction érectile est un critère d'exclusion ; $28,2 \%$ présentent des signes cliniques de prostatite. Le test de Meares et Stamey permet, selon les auteurs, de conclure à l'existence d'une PC bactérienne (à germes aérobies ou Ureaplasma Urealyticum) chez $47,8 \%$ des patients (contre $9 \%$ dans le groupe contrôle), et inflammatoire chez $8,7 \%$. 
En 2006 Shamloul et al. (Egypte) [24] comparent 153 hommes de 29 à 51 ans présentant une éjaculation prématurée ${ }^{\star}$ à un groupe contrôle de 100 hommes avec une méthodologie comparable à celle de Screponi et al. : $12,4 \%$ présentent des signes cliniques de prostatite. Le test de Meares et Stamey permet, selon les auteurs, de conclure à l'existence d'une PC bactérienne chez $53,6 \%$ des patients, et inflammatoire chez 9,8\%. La différence avec le groupe contrôle est significative.

Selon ces deux études une évaluation prostatique, clinique et microbiologique, est recommandée lors d'éjaculation prématurée.

\section{CONCLUSION}

Les PC et SDPC ont un impact sur la qualité de vie et la fonction sexuelle, mais actuellement les données épidémiologiques sont encore limitées. La physiopathologie des dysfonctions sexuelles reste par ailleurs imprécise. Une implication psychologique est très probable mais beaucoup d'incertitudes persistent sur d'autres mécanismes éventuellement en cause. La généralisation de la classification du NIH et l'utilisation de questionnaires validés devraient faciliter la recherche dans ce domaine mais la question est d'autant plus difficile que les mécanismes à l'origine des PC et SDPC ne sont eux-mêmes pas parfaitement élucidés.

\section{Note :}

* L'éjaculation prématurée est définie par un temps IELT (Intravaginal ejaculation latency time) inférieur à $2 \mathrm{mn}$ lors de plus de $50 \%$ des relations sexuelles depuis plus de 6 mois avec une relation hétérosexuelle stable depuis plus d' 1 an.

\section{RÉFÉRENCES}

1. BERGHUIS J.P., HEIMAN J.R., ROTHMAN I., BERGER R.E.: Psychological and physical factors involved in chronic idiopathic prostatitis. J. Psychosom. Res., 1996, 41 : 313-325.

2. CHEAH P.Y., LIONG M.L., YUEN K.H. et al. : Chronic prostatitis, symptom survey with follow-up clinical evaluation. Urology, 2003, 61 : 60-64.

3. COLLINS M.M., MEIGS J.B., BARRY M.J. ET AL. : Prevalence and correlates of prostatitis in the health professionals followup study cohort. J. Urol., 2002, $167:$ 1363-1366.

4. COLLINS M.M., STAFFORD R.S., O'LEARY M.P., BARRY M.J. : How common is prostatitis ? A national survey of physician visits. J. Urol., 1998, $159: 1224-1228$.

5. DANIELS N.A., EWING S.K., ZMUDA J.M., WILT T.J., BAUER
D.C for the osteoporotic fractures in men research group : Correlates and prevalence of prostatitis in a large communitybased cohort of older men. Urology, 2005, 66 : 964-970.

6. DRACH G.W., FAIR W.R., MEARES E.M., STAMEY T.A. : Classification of benign diseases associated with prostatic pain: prostatitis or prostatodynia ? J. Urol., 1978, $120: 266$.

7. GONEN M., KALKAN M., CENKER A., OZKARDES H : Prevalence of premature ejaculation in Turkish men with chronic pelvic pain syndrome. J. Androl., 2005, 26 : 601-603.

8. KELTIKANGAS-JARVINEN L., JARVINEN H., LEHTONEN T. : Psychic disturbances in patients with chronic prostates. Ann. Clin. Res., 1981, $13: 45-49$.

9. KU J.H., KIM M.E., LEE N.K., PARK Y.H. : The prevalence of chronic prostatitis-like symptoms in young men: a community-based survey. Urol. Res., 2001, 29 : 108-112.

10. LIANG C.Z., ZHANG X.J., HAO Z.Y., SHI H.Q., WANG K.X.: Prevalence of sexual dysfunction in Chinese men with chronic prostatitis. Br. J. Urol. Int., 2006, $93: 568-570$.

11. LITWIN M.S., MCNAUGHTON-COLLINS M., FOWLER F.J. JR, ET AL. and Chronic Prostatitis Collaborative Research Network : The National Institutes of Health chronic prostatitis symptom index : development and validation of a new outcome measure. J. Urol., 1999, $162: 369-375$.

12. LUTZ M.C., ROBERTS R.O., JACOBSON D.J., MCGREE M.E., LIEBER M.M., JACOBSEN S.J. : Cross-sectional associations of urogenital pain and sexual function in a community based cohort of older men : Olmsted county, Minnesota. J. Urol., 2005, 174 : 624-628.

13. MEARES E.M., STAMEY T.A. : Bacteriologic localization patterns in bacterial prostatitis and urethritis. Invest. Urol., $1968,5: 492-518$.

14. MEHIKA., HELLSTROM P., LUKKARINEN O., SARPOLAA., JARVELIN M. : Epidemiology of prostatitis in Finnish men: a population-based cross-sectional study. Br. J. Urol. Int., 2000, $86: 443-448$.

15. MEHIK A., HELLSTROM P., SARPOLA A., LUKKARINEN O., JARVELIN M.R. : Fears, sexual disturbances and personality features in men with prostatitis : a populationbased cross-sectional study in Finland. Br. J. Urol. Int., 2006, $88: 35-38$.

16. National Institutes of Health Summary Statement : National Institutes of Health/National Institute of Diabetes and Digestive and Kidney Disease workshop on chronic prostatitis. Bethesda, December 1995.

17. NICKEL J.C., DOWNEY J., HUNTER D., CLARK J. : Prevalence of prostatitis-like symptoms in a population based study using the National Institutes of Health chronic prostatitis symptom index. J. Urol., 2001, $165: 842-845$.

18. NICKEL J.C., ELHILALI M., VALLANCIEN G., ALF-ONE Study Group : Benign prostatic hyperplasia (BPH) and prostatitis : prevalence of painful ejaculation in men with clinical BPH. Br. J. Urol. Int., 2005, 95 : 571-574

19. NICKEL J.C., TEICHMAN J.M., GREGOIRE M., CLARK J., DOWNEY J. : Prevalence, diagnosis, characterization, and treatment of prostatitis, interstitial cystitis, and epididymitis in 
outpatient urological practice : the Canadian PIE Study. Urology, 2005, 66 : 935-940.

20. RIZZO M., MARCHETTI F., TRAVAGLINI F., TRINCHIERI A., NICKEL J.C. : Prevalence, diagnosis and treatment of prostatitis in Italy : a prospective urology outpatient practice study. Br. J. Urol. Int., 2003, 92 : 955-959.

21. ROBERTS R.O., JACOBSON D.J., GIRMAN C.J., RHODES T., LIEBER M.M., JACOBSEN S.J. : Prevalence of prostatitislike symptoms in a community based cohort of older men. J. Urol., 2002, 168 : 2467-2471.

22. ROBERTS R.O., LIEBER M.M., RHODES T., GIRMAN C.J., BOSTWICK D.G., JACOBSEN S.J. : Prevalence of a physician-assigned diagnosis of prostatitis : the Olmsted County Study of Urinary Symptoms and Health Status Among Men. Urology, 1998, $51: 578-584$

23. SCREPONI E., CAROSA E., DI STASI S.M., PEPE M. CARRUBA G., JANNINI E.A. : Prevalence of chronic prostatitis in men with premature ejaculation. Urology, 2001, 58 : 198202.

24. SHAMLOUL R., EL-NASHAAR A. : Chronic prostatitis in premature ejaculation: a cohort study in 153 men. J. Sex. Med., 2006, $3: 150-154$.

25. TAN J.K., PNG D.J., LIEW L.C., LI M.K., WONG M.L. : Prevalence of prostatitis-like symptoms in Singapore : a population-based study. Singapore Med. J., 2002, 43 : 189193.

Manuscrit reçu : septembre 2006 ; accepté septembre 2006.

\begin{abstract}
Chronic prostatitis, chronic pelvic pain syndrome and sexual dysfunction

Dominique DELAVIERRE

In 1995, the NIH (National Institutes of Health, USA) proposed a new classification of chronic prostatitis (CP), no longer considered in the strict framework of the prostate, but based on the concept of pelvic pain. This classification introduced the term chronic pelvic pain syndrome (CPPS). The definition of this syndrome indicates that pain is sometimes associated with sexual disorders.

Many surveys have demonstrated the considerable prevalence of CP/CPPS and have confirmed the impact of these diseases on quality of life, but only limited epidemiological data concerning the links between CP/CPPS and sexuality are available at the present time.

The pathophysiology of sexual dysfunction associated with CP/CPPS (alteration of desire, erectile dysfunction and premature ejaculation) also remains poorly elucidated. A psychological factor is very probably involved, but many uncertainties persist concerning the other mechanisms possibly involved.
\end{abstract}

Key-Words: prostatitis, pelvic pain, sexual dysfunction, erectile dysfunction, premature ejaculation 